\title{
Application of Jacobian defined direct interaction coefficient in DRGEP-based chemical mechanism reduction methods using different graph search algorithms
}

\author{
Yulin Chen*, Jyh-Yuan Chen \\ Department of Mechanical Engineering, University of California Berkeley, Berkeley, CA 94720, USA \\ *Corresponding author: Yulin Chen \\ yulinchen@berkeley.edu \\ Tel: 1-510-837-8238 \\ 246 Hesse Hall, University of California Berkeley, \\ Berkeley, CA 94720, USA
}

Submitted for full-length article with black and white printing 


\section{Abstract}

The graph search based approaches, such as the directed relation graph (DRG) and DRG with error propagation (DRGEP) methods, are efficient as the first-cut reduction for large detailed chemical mechanisms. In this study, the DRGEP-based methods are further improved by using a Jacobian for evaluating direct interaction coefficient (DIC). Both the Dijkstra's algorithm and the AStar algorithm are studied to assess the effect of graph search algorithms on the performance of DIC using the Jacobian implementation. Additionally, the target search algorithm (TSA) is used for exploring the further reduction potential by initializing with the skeletal mechanisms generated by the graph search methods. High temperature methane and low temperature n-heptane auto-ignition are selected as the testing conditions. For methane with high temperature conditions, the Jacobian DIC with the AStar algorithm has the best performance with $31 \%$ species less compared to the previously defined DIC with the Dijkstra's algorithm for the $10 \%$ limited error. By restarting TSA initialized with the skeletal mechanisms generated by graph search methods and TSA, an identical 19-species skeletal mechanism is generated. For nheptane with NTC region conditions, the Jacobian DIC enhances both the two graph search algorithms as well as the methane skeletal reduction. With the combination of the proposed Jacobian DIC and the AStar algorithm, the n-heptane skeletal mechanism generated by repeatedly restarting TSA is $16 \%$ smaller ( 20 species less) than the one purely developed from multi-round TSA for the $10 \%$ error. The validation of $n$-heptane laminar flame speed against detailed mechanism shows that the skeletal mechanisms developed by the DRGEP-based approaches can retain the chemistry of laminar flames by targeting only 30 cases near NTC regions.

Key Words: DRGEP; Sensitivity; Skeletal mechanism; Dijkstra; A-star 


\section{Nomenclature}

DFS: depth first search

DIC: direct interactive coefficient

DRG: directed relation graph

DRGEP: directed relation graph with error propagation

GIC: global interactive coefficient

NTC: negative temperature coefficient

PIC: path interactive coefficient

TSA: target search algorithm

$\varepsilon:$ cutoff tolerance in graph search methods

$t_{\text {ign }}:$ auto-ignition delay time

\section{Introduction}

Since numerical simulations are routinely used in assisting designs of efficient combustion devices, kinetic modeling plays an essential role in simulating combustion processes. However, comprehensive detailed reaction mechanisms covering a wide range of conditions can have thousands of species for heavy fuels [1, 2], which are prohibitively expensive for large-scale three-dimensional computations in terms of memory and CPU time. Additionally, stiffness is another issue when wide ranges of time scales and nonlinear coupling among species exist in the detailed mechanisms. For these reasons, there is great interest in reducing the size of the kinetic model while maintaining sufficient species and reaction steps to adequately reproduce relevant physical phenomena at target conditions [3].

Since the Directed Relation Graph (DRG) method was first proposed a decade ago [4], approaches based on graph search algorithms have received significant attention to develop skeletal mechanisms. DRG and its derivatives are useful for the first-step reduction of large detailed mechanisms due to the efficient features of this type of methods. Additionally, the skeletal mechanisms can be used with analytical Jacobian approaches [5, 6] for efficient computations. However, the efficient characteristic requires that the removal of species in DRG-based methods 
must be accurate enough so that the resulting skeletal mechanism can be as small as possible to satisfy a fixed relative error. Past experiences revealed that only one improper removal of important species would cause the relative error to increase rapidly. Therefore, two notable approaches have been developed on the basis of DRG: (1) Path Flow Analysis (PFA) [7] and (2) DRG with Error Propagation (DRGEP) [8]. In the original DRG, the Direct Interaction Coefficient (DIC) is a pair-wise relation between a pair of two species. The PFA redefines the DIC by considering the production and consumption fluxes separately, and uses the first (A-B) and second generations (A-M-B) for calculating the DIC. The DRGEP modifies the DRG method by considering the path dependence of a target species on others, and take the path product that is searched by the chosen graph search algorithm as the Global Interaction Coefficient (GIC) for each species. DRGEP assumes that the reduction error decays geometrically along the graph searching paths.

The Jacobian is widely applied in chemical kinetics including the solution of stiff ODE, the calculation of local sensitivities and the time-scale analysis [9]. Among them, the local sensitivity calculation is an accurate way to define coupled species from the Jacobian matrix element magnitude [10], and has been employed to identify redundant species [11-13] and reactions [14] in chemical mechanism reduction approaches. Recently, the Jacobian has been used in development of dynamic adaptive chemistry [15] and a linearized error propagation model for skeletal mechanism reduction [16], showing its usefulness in providing key information. However, as a key element in the graph search methods, almost all the DICs are defined by the contribution of production rate from one species to the other. Although there are graph search approaches with the name of DRG-aided Sensitivity Analysis (DRGASA) [17], DRGEP and Sensitivity Analysis (DRGEPSA) [18] and others [19, 20], they are combined methods to check the redundant species by running simulations instead of involving the calculation of sensitivities. For another key factor which is the graph search algorithm, Niemeyer and Sung [21] developed a DRGEP-based method with the Dijkstra's algorithm [22], and compared it with other algorithms, such as Depth-First-Search (DFS) and Breadth-First-Search (BFS) [23, 24] considering the calculation of GIC in DRGEP as a single-source shortest path problem. The results showed that the DRGEP-Dijkstra method is superior to the other algorithms. Thus the graph search algorithms can play an important role to improve the performance 
of DRGEP-based approaches. For the DIC, almost all the graph search approaches focused on the improvements of the definition of production rate contribution from one species to the other [8, 25-27].

In this study, high-temperature methane auto-ignition using the GRI-3.0 mechanism [28] and low-temperature nheptane auto-ignition using the Lawrence Livermore National Lab (LLNL) v3.1 [2] mechanism are selected to be the testing cases. The Jacobian DIC, which employs a semi-normalized local sensitivity coefficient is defined and applied to the DRGEP-based methods. The Dijkstra's algorithm [22] and the AStar algorithm [29] are used to study the effect of graph search algorithms on the Jacobian DIC implementation. Then the Target Search Algorithm (TSA) [30] is applied to compare the further reduction potential by initializing with the skeletal mechanisms developed by graph search methods.

\section{DRG and DRGEP methodology}

In DRG [25] and DRGEP [8] methods, the DICs are related to $r A G$ and $r A B D R G E P$ defined below to measure the relative importance of species B in the chemical activities of species A. These two parameters in DRG and DRGEP are calculated by:

$r A B D R G=i=1 n R v A, i R i \delta B i i=\ln R \nu A, i R i$

$r A B D R G E P=i=1 n R v A, i \operatorname{Ri} \delta \operatorname{Bimax}(P A, C A)$,

where

$$
\begin{aligned}
R i & =R f, i-R b, i \\
& =k f, i j=1 n r, i[C j] v i, j^{\prime}-k b, i j=1 n p, i C j v i, j^{\prime \prime}, \\
P A & =i=1 n R \max (0, v A, i \omega i), \\
C A & =i=1 n R \max (0,-v A, i \omega i),
\end{aligned}
$$


$v i, j=v i, j^{\prime \prime}-v i, j^{\prime}$

The sequence of $\mathrm{AB}$ denotes the direction from species $\mathrm{A}$ to $\mathrm{B}$, which means $\mathrm{A}$ is dependent on $\mathrm{B}$. The symbols $R_{i}, R_{f, i}$ and $R_{b, i}$ are the net, forward and backward reaction rates of the $i$-th reaction, where $v i, j, v i, j{ }^{\prime}$ and $v i, j{ }^{\prime \prime}$ are the net, reactant and product side stoichiometric coefficients of the $j$-th species and the $i$-th reaction, respectively. $C_{j}$ is the molar concentration of species $j . k_{f, i}$ and $k_{b, i}$ are the forward and backward rate coefficients in the $i$-th reaction. $n_{r, i}$ and $n_{p, i}$ are the reactant and product numbers in reaction $i . n_{R}$ is the total number of reactions in the mechanism. $\delta B i$ equals to 1 if the $i$-th reaction involves species $\mathrm{B}$, otherwise it equals to 0 .

In the DRGEP-based method, the importance of path is included by calculating the Path Interaction Coefficient (PIC), $r T B, p$, which is the product of DICs between the target species $\mathrm{T}$ (such as fuel and oxidizer) and the species of interest $\mathrm{B}$ along the path $p$ connecting them:

$r T B, p=j=1 n-1 r S j S j+1 D R G E P$,

where $n$ is the number of species along the path $p . S_{j}$ is the species along the path $p$ by the direction from $T$ to B. As such the error propagation method will assign smaller PICs to the species far from the starting species in a long path since DICs are less than 1. After searched by different graph search algorithms, which will be discussed later, the maximum value of $r T B, p$ will be called $R T B$ corresponding to the assigned location of $\mathrm{B}$ by the searching. Finally, by repeating the proceeding process for all the target species of the preset group $\{X\}$, the Global Interactive Coefficient (GIC), $R B$, is determined for species B:

$R_{B}=\max _{T \in\{X\}} R_{T B}$

Construction of a skeletal mechanism is based on DICs $(=r A B D R G)$ in DRG and on GICs in DRGEP which are compared to a preset cutoff tolerance $(\varepsilon)$. In DRG, after exploring all the species by the chosen search algorithm which uses DICs as a guide, the species without directed edge to any other species will be removed from the detailed mechanism along with its associated reactions. In DRGEP, species with GIC $<\varepsilon$ are removed from the 
species list which is created by searching with a descending order of GICs. In this study, the DICs of Eqs. (1) and (2) are used for the non-Jacobian defined DRG and DRGEP-based methods, respectively.

\section{Jacobian DIC formulation}

The most frequently used form of Jacobian is the first-order local sensitivity coefficient that indicates how a target variable changes as a consequence of a change in a parameter by a small amount, with all the other parameters fixed at their nominal values. In this study, a semi-normalized sensitivity coefficient is calculated as the Jacobian matrix element, which is defined as:

$$
\begin{aligned}
J i, j= & Y j \partial \partial Y j d Y i d t \\
& =Y j W i \rho \partial \omega i \partial Y j \\
= & Y j W i \rho \omega i P, T, Y j+\Delta Y j-\omega i P, T, Y j \Delta Y j
\end{aligned}
$$

$Y i$ and $Y j$ are the mass fraction of the $\mathrm{i}$-th and $\mathrm{j}$-th species. $W i$ and $\omega i$ are the molecular weight and the molar production rate of the $i$-th species, respectively, and $\rho$ is the density. The local sensitivity shown in Eq. (11) is computed numerically. The Jacobian DIC is defined in Eq. (12), which is the ratio between the absolute Jacobian matrix element and the maximum absolute values among the elements in the same row. Physically, through changing the mass fraction of the species $\mathrm{B}$, the perturbed production rate of the species $\mathrm{A}$ is resulted from a globally synergy effect by all the related reactions that species B are involved. In comparison, the original DICs shown in Eqs. (1) and (2) only define the local interactions by considering the reactions including both species A and $\mathrm{B}$.

$r A B J A C=J A, B \max 1 \leq j \leq n s p(J A, j, 10-40)$

\section{Graph search algorithms in DRGEP-based methods}

In Figure 1, a 5-node directed graph is plotted as an example with the DIC value for each edge. The nodes represent the species in a detailed reaction mechanism. The arrow from species $\mathrm{X}$ to species $\mathrm{Y}$ denotes that $\mathrm{X}$ is dependent on Y. The searching processes of the three graph search algorithms are explained through the DRGEP- 
based methods to reveal their effects on the species list ranking. Node A is the pre-defined target species, such as fuel and oxidizer, to be kept in the skeletal mechanisms. Because Niemeyer and Sung [21] showed the incompatibility performance between the DFS algorithm [31] and the DRGEP-based methods, the DFS algorithm is only used in the original DRG method and won't be shown for the searching process in this section.

Figure 1. A 5-node directed graph with the DIC value for each edge.

\subsection{Dijkstra's algorithm}

The Dijkstra's algorithm $[22,31]$ is a shortest path searching algorithm. The purpose of calculating GIC in DRGEP-based methods is to determine the maximum product value of DIC between the target species and the species of interest in all possible paths. As an important feature, the Dijkstra's algorithm is guided by PIC considering all the unexplored species. In Figure 2, the searching process using the Dijkstra's algorithm is shown for the directed graph in Figure 1. The numbers inside the brackets and parentheses indicate the searching sequences and the GICs $(R B=R T B$ due to one target), respectively. Starting from the target Node A, Node $\mathrm{B}$ is reached first due to the highest PIC among all the species related to A. Then Node D is recorded due to its higher DIC $\left(r_{B D}=0.9\right)$ than the other unexplored species related to B $\left(r_{B C}=0.4\right.$ and $\left.r_{B E}=0.1\right)$. The word "recorded" here means the Node D has not been flagged as a "searched" node because its PIC ( $\left.r_{A B D, p}=0.63\right)$ needs to be compared with the PICs of the other unexplored species related to A $\left(r_{A C, p}=0.5, r_{A D, p}=0.4\right.$ and $\left.r_{A E, p}=0.6\right)$. The subscript "ABD" is used to be distinguished from the path involves only A and D. Since the $r_{A B D, p}$ is the largest among them, Node

$\mathrm{D}$ is searched. Then $r_{A B D E, p}=0.126$ is recorded and compared with the unexplored $r_{A C, p}=0.5$ and $r_{A E, p}=0.6$. Thus Node $E$ is searched, followed by Node $C$. Therefore, the species list is $B(0.7)>D(0.63)>E(0.6)>C(0.5)$.

Figure 2. Searching processes and results of the Dijkstra's algorithm through a DRGEP-based method.

\subsection{AStar algorithm}


The AStar (A*) search [29] is a best-first-search algorithm widely used in path-finding [32]. A key feature is that the AStar is an aim-guided algorithm with a heuristic estimation function. Depending on the desired outcome, the heuristic estimation can be defined by different functions. For mechanism reduction, this heuristic estimation function is related to the importance of the searched species to a destination species. That is, in addition to the target species, a group of destination species is selected to be included in the skeletal mechanism. After the search, the GIC $(R B)$ in Eq. (8) is modified as Eq. (13) by multiplying the heuristic estimation term $R B D$, which is the

PIC after graph search between B and the destination $D$. The destination species is defined with a preset group $\{Y\}$ consisting of products, key intermediate species or pollutants, such as $\mathrm{CO}_{2}, \mathrm{H}_{2} \mathrm{O}, \mathrm{HO}_{2}$ and NO . Through combinations between the target group $\{X\}$ and destination group $\{Y\}$, the searching is conducted for all paths to determine $R B$. Eq. (14) is the PIC to be compared in different paths during the searching. In the work of Zhao et al. [27], the concept of shortest path and Betweenness Centrality (BC) is proposed, which is similar to the concept of AStar algorithm by introducing the pair of source and target nodes. In this study, the Dijkstra's algorithm is used for $r_{T B, p}$ and $r_{B D, p}$ to find the "crucial paths". Therefore, the AStar algorithm can be viewed as an embededDijkstra's algorithm. In Figure 3, the searching process and results are shown for the AStar algorithm in a DRGEP-based method. Species E is defined as the destination to be kept in the skeletal mechanisms. The dashed arrows in the plot represent the heuristic estimation processes. The results show that, smaller GICs (numbers in parentheses) are calculated in DRGEP-AStar than DRGEP-Dijkstra because product of double paths is considered in the AStar algorithm. For instance, GIC for B is 0.126 in comparison with 0.7 by the Dijkstra's algorithm. These smaller values won't affect the fairness of search since species are removed on the basis of relative ranking in comparison to $\varepsilon$. In the current example, because $\mathrm{A}$ and $\mathrm{E}$ are preset to be kept, the species list with descending order of GIC is $\mathrm{C}(0.15)>\mathrm{B}(0.126)=\mathrm{D}(0.126)$, which is different from the species ranking by the Dijkstra's algorithm because $\mathrm{D}$ and $\mathrm{C}$ are more important to $\mathrm{E}$ than $\mathrm{B}$ during the search by AStar algorithm.

$$
R_{B}=\max _{T \in\{X\}, D \in\{Y\}}\left(R_{T B} R_{B D}\right)
$$

$r B D, p=j=1 m-1 r S j S j+1 D R G E P$ 
Figure 3. Searching process and result of the AStar algorithm through a DRGEP-based method.

\section{Target search algorithm (TSA)}

In order to assess the performances and further reduction potential of DRG and DRGEP-based methods, the Target Search Algorithm (TSA) [30, 33] is used as a reference. The TSA is a trial and error method to develop skeletal mechanisms and it has been demonstrated to generate accurate skeletal mechanisms. However, TSA becomes expensive when the starting mechanism is large $[30,34]$. In the TSA, the species in the detailed mechanism are tested for removal in ascending order by their maximum mole fractions. After the removal of each test species and associated reactions, the relative error of the new skeletal mechanism is compared to the original mechanism by running flame or kinetics codes at each target condition. If the relative errors do not exceed predefined thresholds, the tested candidate will be removed from the mechanism; otherwise, the species and reactions are re-inserted into the mechanism. The above process is repeated for all species. TSA becomes time-consuming for large detailed mechanisms, especially when there are flame speed target cases. Therefore, the graph search methods can be employed as the first step of the reduction process. Then the TSA is applied once the graph search method can't satisfy the relative error threshold. This reduction strategy would benefit from a smallest starting mechanism generated by DRG or DRGEP-based methods with good accuracy.

\section{Results and discussion}

The application of Jacobian DIC in DRGEP-based methods is implemented with the Dijkstra's algorithm (Dijkstra-JAC) and the AStar algorithm (AStar-JAC), which are compared with the DRGEP-Dijkstra [21], the DRGEP-AStar and the original DRG [4] for high-temperature methane and low-temperature n-heptane autoignition. The maximum relative error between the predicted results of detailed and skeletal mechanisms is defined in Eq. (15), where $x$ denotes a key feature to be computed accurately by the skeletal mechanism. $Z$ represents the collection of all the target cases of interest.

סskel=maxk $\in$ Zxdetk-xskelkxdetk 
In this study, an in-house Well-Mixed Reactor (WMR) model [35] is used for evaluating the relative error of autoignition delay time $\left(t_{\text {ign }}\right)$ with the threshold of $10 \%$ by assuming adiabatic constant volume calculation. For the inputs of chemical state samples in WMR model to the graph search approaches, six temperature points at temperature increments of $20 \mathrm{~K}, 50 \mathrm{~K}, 150 \mathrm{~K}, 300 \mathrm{~K}, 550 \mathrm{~K}$ and $1000 \mathrm{~K}$ over the initial temperature of the target cases are selected.

\subsection{Skeletal reduction of methane auto-ignition under high temperatures}

The GRI-3.0 [28] mechanism with 53 species and 325 reactions is used for computing methane auto-ignition. Although GRI-3.0 is a relatively small mechanism compared to those of heavy fuels, the single-stage ignition is a good candidate for comparing the accuracy among the five methods under high temperature conditions. Twenty cases are run with conditions in the range of $\mathrm{T}=\{1100 \mathrm{~K}, 1300 \mathrm{~K}\}, \mathrm{P}=\{10 \mathrm{~atm}, 40 \mathrm{~atm}\}$ and equivalence ratio $(\varphi)=\{0.5,0.8,1.0,1.2,1.5\}$. The target species in the DRGEP-AStar related methods consist of $\mathrm{CH}_{4}, \mathrm{O}_{2}$, and $\mathrm{N}_{2}$, and the destination species contain $\mathrm{CO}_{2}, \mathrm{H}_{2} \mathrm{O}$ and $\mathrm{N}_{2}$. Nine combinations can be made between the two groups but there are eight paths to be searched because the path of $\mathrm{N}_{2}-\mathrm{N}_{2}$ is not needed. The target species in the DRG and DRGEP-Dijkstra methods consist of $\mathrm{CH}_{4}, \mathrm{O}_{2}, \mathrm{~N}_{2}, \mathrm{CO}_{2}$, and $\mathrm{H}_{2} \mathrm{O}$. By setting different values of the cutoff level $\varepsilon$, skeletal mechanisms with different sizes can be developed. In Figure 4, the maximum relative error is plotted against the species number of the skeletal mechanism generated by various graph search approaches. The details of the species number, reaction number, maximum relative error of graph search methods, TSA and TSA-restart are summarized in Table 1. The red dashed line in Figure 4 denotes the $10 \%$ cutoff level that is acceptable for the subsequent reduction methods. If the maximum relative error is larger than $10 \%$ for the next species removal, the number of species and reactions, and the maximum relative error of the current skeletal mechanism are recorded in the second to fourth columns of Table 1, respectively. As seen in Figure 4 and Table 1, the DRGEP-AStar with Jacobian DIC is found to generate the smallest size of the skeletal mechanism (22 species) among the graph search approaches. Overall, the Jacobian DIC is seen to decrease the size of skeletal mechanism generated by the DRGEP methods using the same graph search algorithms. The AStar algorithm is found to perform better than the Dijkstra' algorithm with the same DICs. Although the TSA method is more time consuming than the graph search methods, the detailed mechanism can be reduced to 19 species satisfying the relative error threshold of $10 \%$ by 
one-round running. Since the graph search methods can be used as the first-step reduction for a large mechanism, the TSA is run with the skeletal mechanisms as the initial mechanism shown in the second column of Table 1 until there is no species that can be removed to satisfy the $10 \%$ cutoff level. This exercise checks further reduction potential to the final skeletal mechanisms. The results in the last three columns of Table 1 indicate that the TSArestart running initialized by the graph methods can lead to the identical 19-species skeletal mechanisms with TSA. In Figure 5, the comparison of methane ignition delay time between the detailed mechanism and the final 19-species skeletal mechanism is plotted. From the results, the high temperature region can be accurately predicted by the skeletal mechanism.

Figure 4. The maximum relative error against species number of skeletal mechanism developed by various graph search methods under high temperature conditions. The detailed mechanism is the GRI-3.0 mechanism [28] with 53 species.

\section{Table 1}

Figure 5. Comparison of methane ignition delay time between the detailed mechanism and the final 19-species skeletal mechanisms.

\subsection{Skeletal reduction of n-heptane auto-ignition in NTC regions}

For the graph search methods, the low-temperature combustion, Negative Temperature Coefficient (NTC) region and two-stage ignition, presents challenges to fulfill large scale reduction for a fixed threshold. In practice, the combustion process in engine conditions are mainly under these regions [30]. Therefore, n-heptane, which involves NTC region and two-stage ignition, is chosen as the next test fuel. The detailed mechanism by Lawrence Livermore National Lab (LLNL) v3.1 consisting of 654 species and 2827 reactions [2] was selected for reduction. 
Thirty targets conditions covering the NTC regions are chosen in the range of $\mathrm{P}=\{20 \mathrm{~atm}, 40 \mathrm{~atm}\}, \varphi=\{0.5,1.0$, $2.0\}$ and $\mathrm{T}=\{725 \mathrm{~K}, 775 \mathrm{~K}, 850 \mathrm{~K}, 900 \mathrm{~K}, 950 \mathrm{~K}\}$ shown in Figure 6.

Figure 6. Thirty n-heptane target cases selected for LLNL v3.1 mechanism reduction with NTC regions.

Figure 7 presents the maximum relative error against species number of the skeletal mechanism generated by various graph search approaches and the results are summarized in Table 2 . The red line indicates the $10 \%$ cutoff level. The records of the species number, reaction number, maximum relative error of graph search methods, TSA and TSA-restart are shown in Table 2. From the results in Figure 7 and Table 2, the DRGEP-Dijkstra with Jacobian DIC is found to generate the smallest skeletal mechanism (247 species) with $8 \%$ error among graph search methods. Similar to the conclusion drawn in the high temperature skeletal reduction for methane, the Jacobian DIC is shown to improve the DRGEP-based methods compared to the previously defined. However, with the AStar algorithm, the improvement is small, i.e., 258 species versus 264 species. The one-round TSA can reduce the detailed mechanism to a much smaller size with 183 species satisfying $10 \%$ maximum relative error. Then the further reduction potential is tested by repeatedly running TSA-restart based on the skeletal mechanisms in the second column of Table 2 until there is no species removal for the fixed $10 \%$ cutoff level. The results in the last three columns of Tables 2 suggest that all the graph search methods lead to smaller final skeletal mechanisms than the TSA method alone. The DRGEP-AStar with Jacobian DIC leads to the smallest mechanism of 117 species compared to 139 species of the TSA method by $16 \%$ difference. The restart results with TSA also reveal that DRGEP-AStar leads to a smaller final mechanism than DRGEP-Dijkstra (123 species). The better performance of DRGEP-AStar than the DRGEP-Dijkstra with Jacobian DIC may be fortuitous as both algorithms perform similarly well. Based on the above results, the application of Jacobian DIC makes the Dijkstra's algorithm and AStar algorithm perform better compared to the original DICs.

Figure 7. The maximum relative error against species number of skeletal mechanism developed by various graph search methods in NTC regions. 


\section{Table 2}

In Figures 8 and 9, the ignition delay times of the final n-heptane skeletal mechanisms are compared to those from the detailed mechanism. As seen in the figures, the skeletal mechanisms can match the detailed mechanism very well with the prescribed $10 \%$ error bound. Under some partially premixed engine conditions, the laminar flame speed is of great interest in chemical mechanism reduction [36]. In this study, only 30 target ignition cases covering the NTC regions under high pressures are used for development of various skeletal mechanisms. It would be too costly to include flame speed computation during the development. At the end of the first-step reduction, the skeletal mechanisms from various graph-based approaches are checked for their accuracy in predicting flame speeds using PREMIX [37] before further reductions. Figures 10 and 11 present comparisons of computed flames against experimental data at various initial temperatures and pressures revealing that all the graph-based methods in this study can generate skeletal mechanisms with very high accuracy compared to the detailed mechanism for all the conditions considered. Moreover, the results confirm that despite of their smaller sizes, the skeletal mechanisms generated by using Jacobian DIC retain the accurate predictability for the laminar flame speeds.

Figure 8. Comparison of $n$-heptane ignition delay time between the detailed mechanism and the final skeletal mechanisms for equivalence ratio of $0.5,1.0$ and 2.0 under the pressure of $20 \mathrm{~atm}$.

Figure 9. Comparison of ignition delay time between the detailed mechanism and the final skeletal mechanisms for equivalence ratio of $0.5,1.0$ and 2.0 under the pressure of $40 \mathrm{~atm}$.

Figure 10. Comparison of laminar flame speed between the detailed mechanism and skeletal mechanisms generated by DRGEP-based methods for unburned gas temperature of $298 \mathrm{~K}, 400 \mathrm{~K}$ and $470 \mathrm{~K}$ under the pressure of 1 atm. Symbols are experimental data: triangle: Kumar et al. [38]; square: Davis and Law [39]; circle: Huang et al. [40]; pentagon: Sileghem et al. [41]. 
Figure 11. Comparison of laminar flame speed between the detailed mechanism and skeletal mechanisms generated by DRGEP-based methods for pressure of $1 \mathrm{~atm}, 2 \mathrm{~atm}$ and $5 \mathrm{~atm}$ under the unburned gas temperature of 353 K. Symbols are experimental data: triangle: Kumar et al. [38]; hexagon: Smallbone et al. [42]; star: Kelley et al. [43].

\section{Conclusion}

In this study, a Jacobian-based direct interactive coefficient is proposed and applied to the DRGEP-based method with the Dijkstra's algorithm and the AStar algorithm. Comparisons of resulting skeletal mechanisms are made between the original and newly developed approaches for both the high temperature methane and the low temperature n-heptane auto-ignition. Moreover, the trail-and-error approach called TSA is used to explore the further reduction potential for the graph search methods. The major results and findings are:

- For methane with high temperature conditions, the Jacobian DIC with the AStar algorithm has the best performance with $31 \%$ species less compared to the original DIC with Dijkstra's algorithm for the $10 \%$ limited error. By restarting TSA with the skeletal mechanisms generated by graph search methods, an identical mechanism with 19 species is reached.

- For n-heptane with NTC region conditions, the Jacobian DIC enhances both the two graph search algorithms. With the combination of the proposed Jacobian DIC and the AStar algorithm, the n-heptane skeletal mechanism generated by restarting TSA is $16 \%$ smaller ( $\sim 20$ species less) than the one developed from multi-round TSA for the $10 \%$ error.

- The comparisons of laminar flame speeds among the detailed mechanism and the various skeletal mechanisms developed by the graph-based approaches over a wide range of equivalence ratio, temperature, and pressure indicate that the chosen target cases covering the NTC region are sufficient to retain the chemistry of the laminar flames. Therefore, the DRGEP-based methods with Jacobian DIC are advantageous to be used as the first-step reduction for the subsequent reduction methods such as TSA.

\section{Acknowledgments}


This work at the University of California Berkeley was supported by National Science Foundation and U.S.

Department of Energy under award No. CBET-1258653.

\section{References}

[1] C.K. Westbrook, C.V. Naik, O. Herbinet, W.J. Pitz, M. Mehl, S.M. Sarathy, H.J. Curran, Detailed chemical kinetic reaction mechanisms for soy and rapeseed biodiesel fuels, Combust. Flame 158 (2011) 742-755.

[2] M. Mehl, W.J. Pitz, C.K. Westbrook, H.J. Curran, Kinetic modeling of gasoline surrogate components and mixtures under engine conditions, Proc. Combust. Inst. 33 (2011) 193-200.

[3] T. Lu, C.K. Law, Toward accommodating realistic fuel chemistry in large-scale computations, Prog. Energy Combust. Sci. 35 (2009) 192-215.

[4] T. Lu, C.K. Law, A directed relation graph method for mechanism reduction, Proc. Combust. Inst. 30 (2005) 1333-1341.

[5] K.E. Niemeyer, N.J. Curtis, C.-J. Sung, pyJac: analytical Jacobian generator for chemical kinetics, under review arXiv:1605.03262 [physics.comp-ph] (2016).

[6] F. Perini, E. Galligani, R.D. Reitz, An Analytical Jacobian Approach to Sparse Reaction Kinetics for Computationally Efficient Combustion Modeling with Large Reaction Mechanisms, Energy Fuels 26 (2012) 4804-4822.

[7] W. Sun, Z. Chen, X. Gou, Y. Ju, A path flux analysis method for the reduction of detailed chemical kinetic mechanisms, Combust. Flame 157 (2010) 1298-1307.

[8] P. Pepiot-Desjardins, H. Pitsch, An efficient error-propagation-based reduction method for large chemical kinetic mechanisms, Combust. Flame 154 (2008) 67-81.

[9] T. Turányi, A.S. Tomlin, Analysis of kinetic reaction mechanisms, Springer, Berlin, 2014.

[10] T. Turányi, Sensitivity analysis of complex kinetic systems. Tools and applications, J. Math. Chem. 5 (1990) 203-248.

[11] S. Vajda, P. Valko, T. Turanyi, Principal component analysis of kinetic models, Int. J. Chem. Kinet. 17 (1985) 55-81.

[12] T. Turányi, Reduction of large reaction mechanisms, New J. Chem. 14 (1990) 795-803.

[13] T. Nagy, T. Turányi, Reduction of very large reaction mechanisms using methods based on simulation error minimization, Combust. Flame 156 (2009) 417-428.

[14] T. Turanyi, T. Berces, S. Vajda, Reaction rate analysis of complex kinetic systems, Int. J. Chem. Kinet. 21 (1989) 83-99.

[15] W. Xie, Z. Lu, Z. Ren, L. Hou, Dynamic Adaptive Chemistry via Species Time-scale and Jacobianaided Rate Analysis, Proc. Combust. Inst. DOI information: 10.1016/j.proci.2016.07.105, 2016.

[16] Y. Liu, Y. Wu, Y. Gao, T. Lu, A linearized error propagation model based on Jacobian analysis for skeletal mechanism reduction, 9th US National Combustion Meering, Cincinnati, 2015.

[17] X.L. Zheng, T.F. Lu, C.K. Law, Experimental counterflow ignition temperatures and reaction mechanisms of 1,3-butadiene, Proc. Combust. Inst. 31 (2007) 367-375.

[18] K.E. Niemeyer, C.-J. Sung, M.P. Raju, Skeletal mechanism generation for surrogate fuels using directed relation graph with error propagation and sensitivity analysis, Combust. Flame 157 (2010) 1760-1770.

[19] R. Li, S. Li, F. Wang, X. Li, Sensitivity analysis based on intersection approach for mechanism reduction of cyclohexane, Combust. Flame 166 (2016) 55-65.

[20] A. Stagni, A. Frassoldati, A. Cuoci, T. Faravelli, E. Ranzi, Skeletal mechanism reduction through species-targeted sensitivity analysis, Combust. Flame 163 (2016) 382-393.

[21] K.E. Niemeyer, C.-J. Sung, On the importance of graph search algorithms for DRGEP-based mechanism reduction methods, Combust. Flame 158 (2011) 1439-1443.

[22] E.W. Dijkstra, A note on two problems in connexion with graphs, Numerische Mathmatik 1 (1959) 269-271. 
[23] L. Liang, J.G. Stevens, J.T. Farrell, A dynamic adaptive chemistry scheme for reactive flow computations, Proc. Combust. Inst. 32 (2009) 527-534.

[24] Y. Shi, H.W. Ge, B.J. L., R.D. Reitz, Automatic chemistry mechanism reduction of hydrocarbon fuels for $\mathrm{HCCl}$ engines based on DRGEP and PCA methods with error control, Energy Fuels 24 (2010) 1646-1654.

[25] T. Lu, C.K. Law, On the applicability of directed relation graphs to the reduction of reaction mechanisms, Combust. Flame 146 (2006) 472-483.

[26] Z. Luo, T. Lu, M.J. Maciaszek, S. Som, D.E. Longman, A Reduced Mechanism for HighTemperature Oxidation of Biodiesel Surrogates, Energy Fuels 24 (2010) 6283-6293.

[27] P. Zhao, S.M. Nackman, C.K. Law, On the application of betweenness centrality in chemical network analysis: Computational diagnostics and model reduction, Combust. Flame 162 (2015) 29912998.

[28] G.P. Smith, D.M. Golden, M. Frenklach, N.W. Moriarty, B. Eiteneer, M. Goldenborg, C.T. Bowman, R.K. Hanson, S. Song, W.C. Gardiner, V.V. Lissianski, Z. Qin, available at $<$ http://www.me.berkeley.edu/gri mech/>.

[29] P.E. Hart, N.J. Nilsson, B. Raphael, A Formal Basis for the Heuristic Determination of Minimum Cost Paths, IEEE Trans. Syst. Sci. Cybern. SSC-4 (1968) 100-107.

[30] Y.F. Tham, F. Bisetti, J.-Y. Chen, Development of a Highly Reduced Mechanism for Iso-Octane HCCl Combustion With Targeted Search Algorithm, . J. Eng. Gas Turbines Power 130 (2008) 042804.

[31] S. Dasgupta, C.H. Papadimitriou, U. Vazirani, Algorithms, McGraw-Hill, New York, 2006.

[32] S. Russel, P. Norvig, Artificial Intelligence: A Modern Approach, Pearson, Boston, 2009.

[33] J.-Y. Chen, Automatic Generation of Reduced mechanisms and Their Applications to Combustion Modeling, Trans. Aeronaut. Astronaut. Soc. China 33 (2001) 59-67.

[34] M. Mehl, J.Y. Chen, W.J. Pitz, S.M. Sarathy, C.K. Westbrook, An Approach for Formulating Surrogates for Gasoline with Application toward a Reduced Surrogate Mechanism for CFD Engine Modeling, Energy Fuels 25 (2011) 5215-5223.

[35] J.Y. Chen, R.W. Dibble, J. Kolbu, R. Homma, Optimization of homogeneous charge compression ignition with genetic algorithms, Combust. Sci. and Tech. 175 (2003) 373-392.

[36] G.T. Kalghatgi, Developments in internal combustion engines and implications for combustion science and future transport fuels, Proc. Combust. Inst. 35 (2015) 101-115.

[37] R.J. Kee, F.M. Rupley, E. Meeks, J.A. Miller, CHEMKIN-III: A FORTRAN chemical kinetics package for the analysis of gas-phase chemical and plasma kinetics, Sandia national laboratories report SAND96-8216, (1996).

[38] K. Kumar, J.E. Freeh, C.J. Sung, Y. Huang, Laminar Flame Speeds of Preheated isoOctane/O2/N2 and n-Heptane/O2/N2 Mixtures, J. Propul. Power 23 (2007) 428-436.

[39] S.G. Davis, C.K. Law, Determination of and Fuel Structure Effects on Laminar Flame Speeds of C1 to C8 Hydrocarbons, Combust. Sci. and Tech. 140 (1998) 427-449.

[40] Y. Huang, C.J. Sung, J.A. Eng, Laminar flame speeds of primary reference fuels and reformer gas mixtures, Combust. Flame 139 (2004) 239-251.

[41] L. Sileghem, V.A. Alekseev, J. Vancoillie, K.M. Van Geem, E.J.K. Nilsson, S. Verhelst, A.A. Konnov, Laminar burning velocity of gasoline and the gasoline surrogate components iso-octane, $n$ heptane and toluene, Fuel 112 (2013) 355-365.

[42] A.J. Smallbone, W. Liu, C.K. Law, X.Q. You, H. Wang, Experimental and modeling study of laminar flame speed and non-premixed counterflow ignition of n-heptane, Proc. Combust. Inst. 32 (2009) 12451252.

[43] A.P. Kelley, A.J. Smallbone, D.L. Zhu, C.K. Law, Laminar flame speeds of C5 to C8 n-alkanes at elevated pressures: Experimental determination, fuel similarity, and stretch sensitivity, Proc. Combust. Inst. 33 (2011) 963-970. 


\section{Table 1}

Comparison of methane skeletal mechanism sizes generated by different reduction methods*.

\begin{tabular}{lcccccc}
\hline Method & \# Species & \# Reactions & $\begin{array}{c}\text { Max. rel. error } \\
\text { \# Species }\end{array}$ & \# Reactions & Max. rel. error (\%) \\
& & & $(\%)$ & by TSA-restart & by TSA-restart & by TSA-restart \\
\hline DRG & 45 & 266 & 9.16 & 19 & 79 & 8.97 \\
Dijkstra & 32 & 165 & 9.07 & 19 & 79 & 8.97 \\
AStar & 26 & 138 & 9.16 & 19 & 79 & 8.97 \\
Dijkstra-JAC & 25 & 132 & 2.53 & 19 & 79 & 8.97 \\
AStar-JAC & 22 & 92 & 3.71 & 19 & 79 & 8.97 \\
TSA & 19 & 79 & 8.97 & 19 & 79 & 8.97 \\
\hline
\end{tabular}

* Maximum relative error is set at 10\%. The TSA-restart means keeping running TSA based on the skeletal mechanism in the $2^{\text {nd }}$ column until there is no species removed by TSA. The detailed mechanism used is the GRI3.0 with 53 species and 325 reactions. 


\section{Table 2}

Comparison of n-heptane skeletal mechanism sizes generated by different reduction methods*.

\begin{tabular}{|c|c|c|c|c|c|c|}
\hline Method & \# Species & \# Reactions & $\begin{array}{l}\text { Max. rel. error } \\
(\%)\end{array}$ & $\begin{array}{l}\text { \# Species } \\
\text { by TSA-restart }\end{array}$ & $\begin{array}{l}\text { \# Reactions } \\
\text { by TSA-restart }\end{array}$ & $\begin{array}{l}\text { Max. rel. error (\%) } \\
\text { by TSA-restart }\end{array}$ \\
\hline DRG & 341 & 1745 & 7.88 & 129 & 559 & 9.94 \\
\hline Dijkstra & 273 & 1452 & 5.37 & 127 & 570 & 9.61 \\
\hline AStar & 264 & 1395 & 9.23 & 118 & 528 & 9.84 \\
\hline Dijkstra-JAC & 247 & 1198 & 7.96 & 123 & 536 & 9.58 \\
\hline AStar-JAC & 258 & 1367 & 9.43 & 117 & 519 & 9.62 \\
\hline TSA & 183 & 797 & 9.80 & 139 & 639 & 9.96 \\
\hline
\end{tabular}

* Maximum relative error is set at 10\%. The TSA-restart means keeping running TSA based on the skeletal mechanism in the $2^{\text {nd }}$ column until there is no species removed by TSA. The detailed mechanism used is the LLNL v3.1 with 654 species and 2827 reactions. 


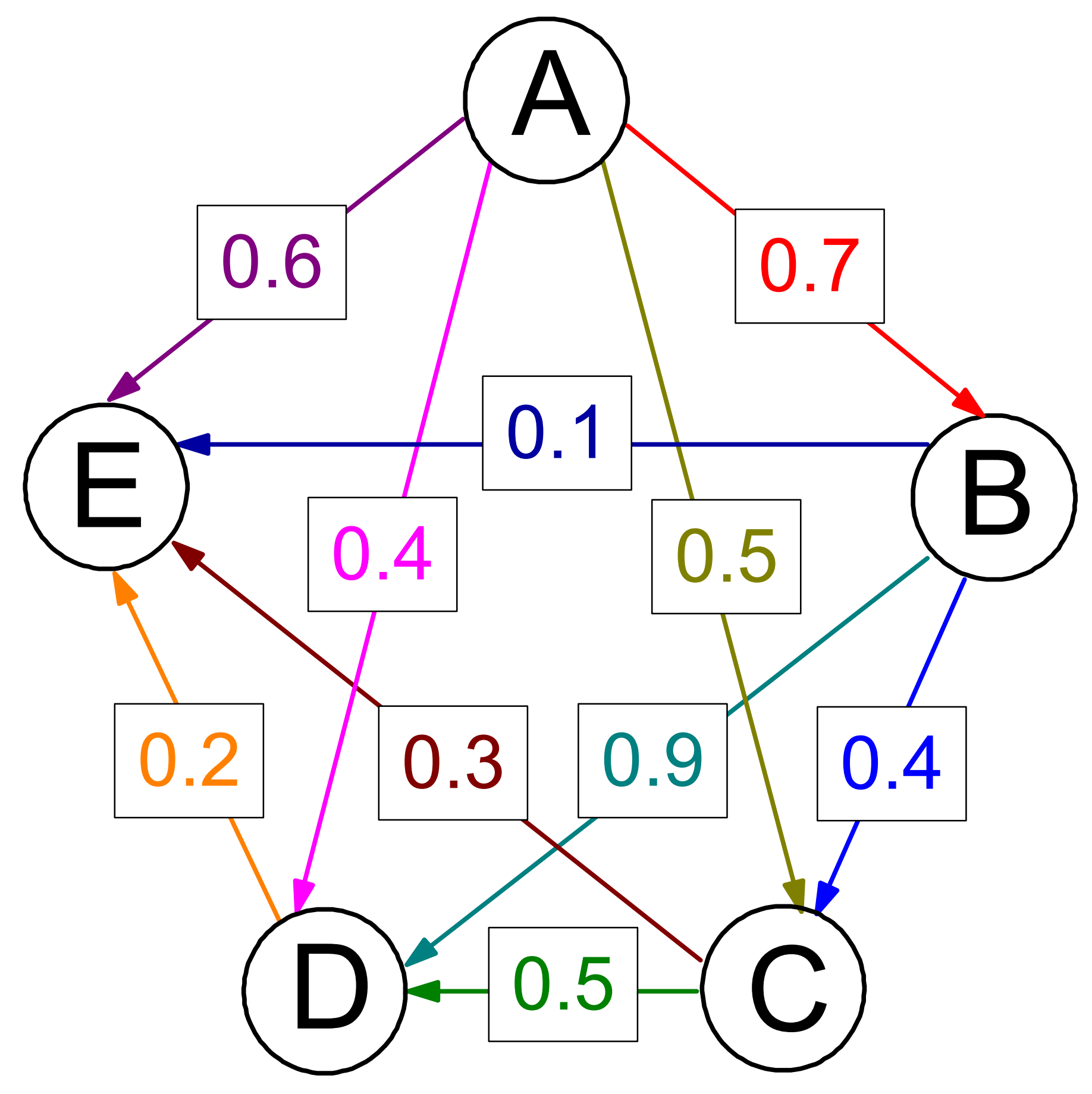

Figure 1 


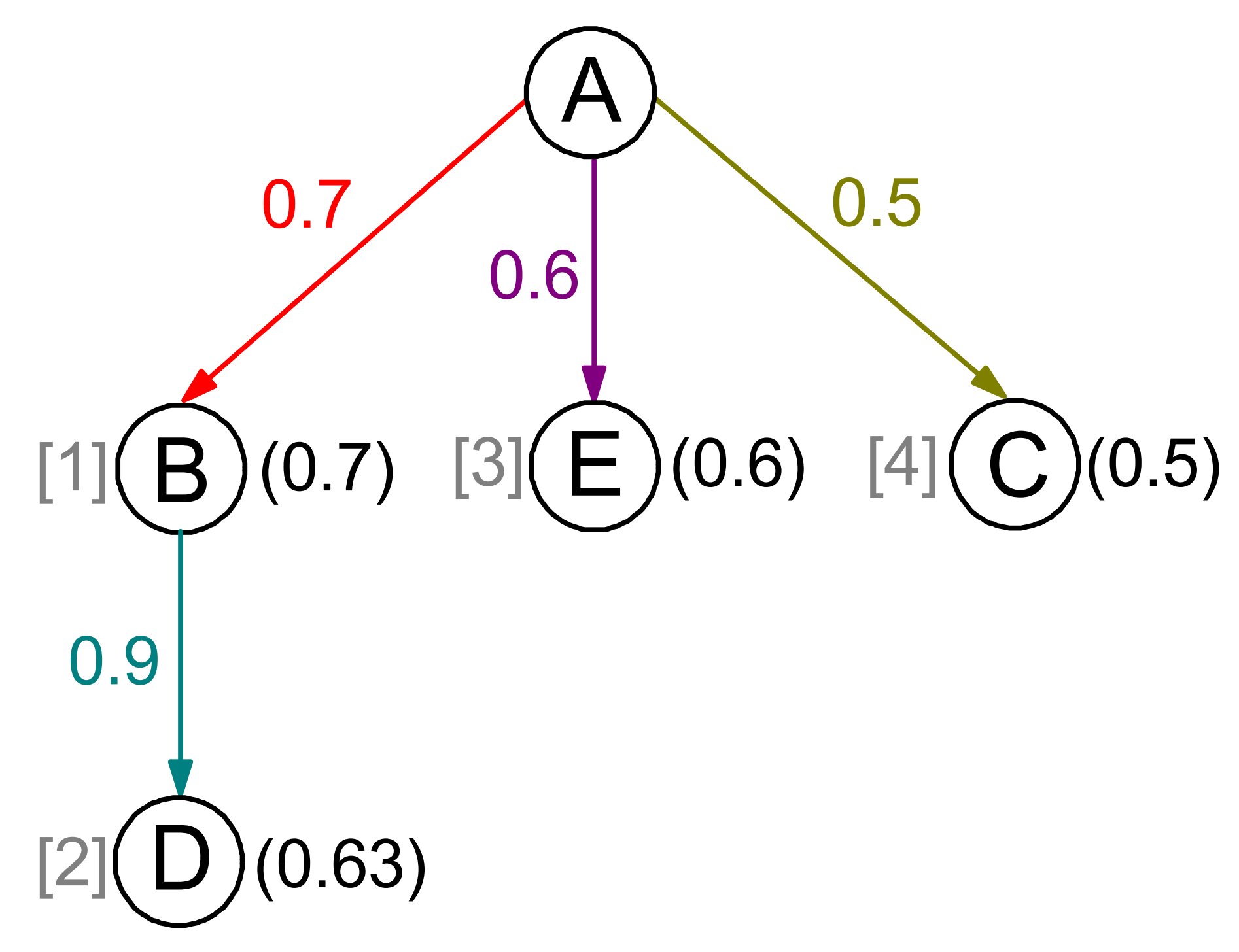

Figure 2

\section{Dijkstra}

Figure 2

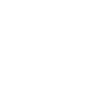

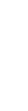

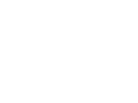




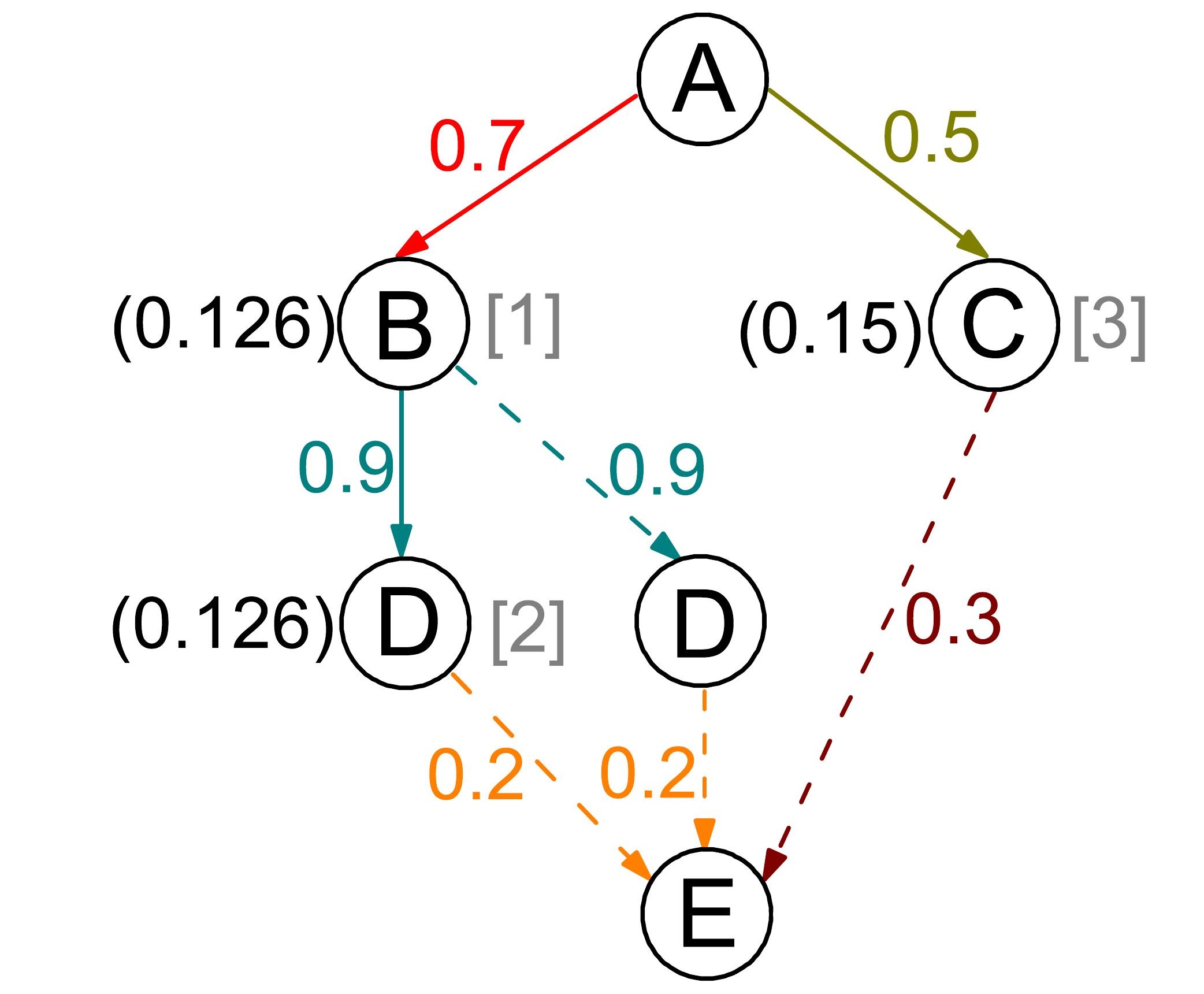

Figure 3

\section{A-star}

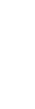


Figure 4

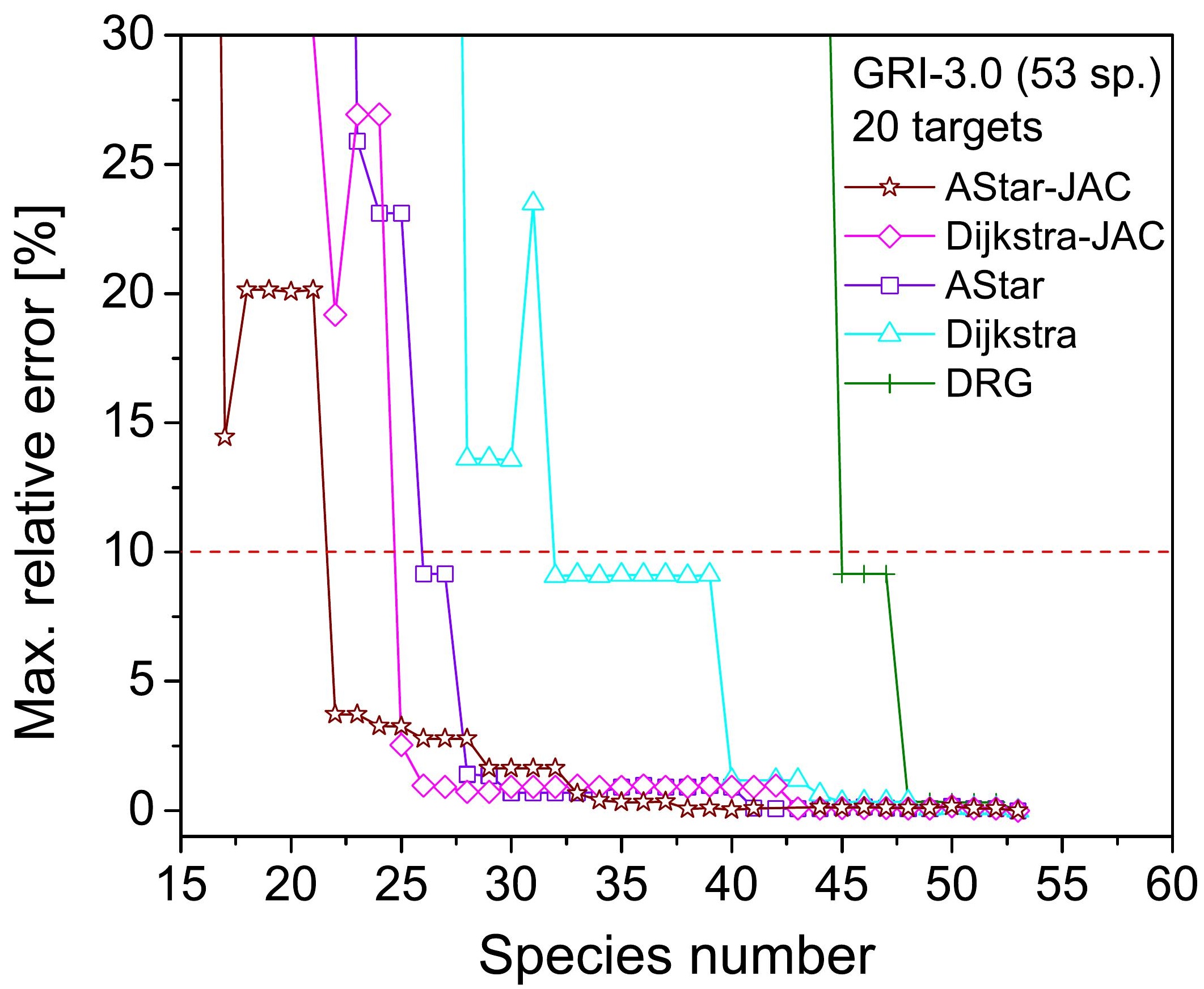




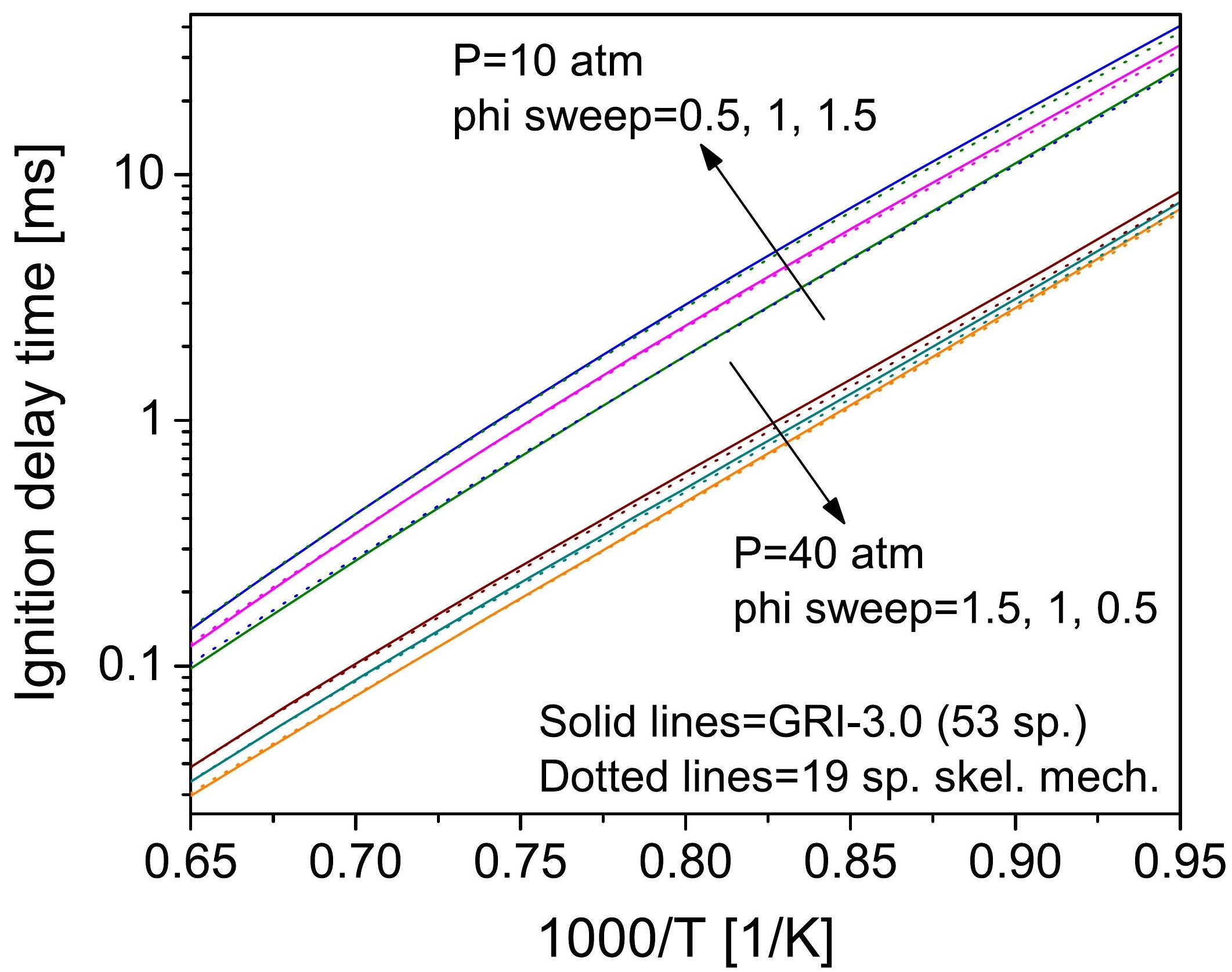




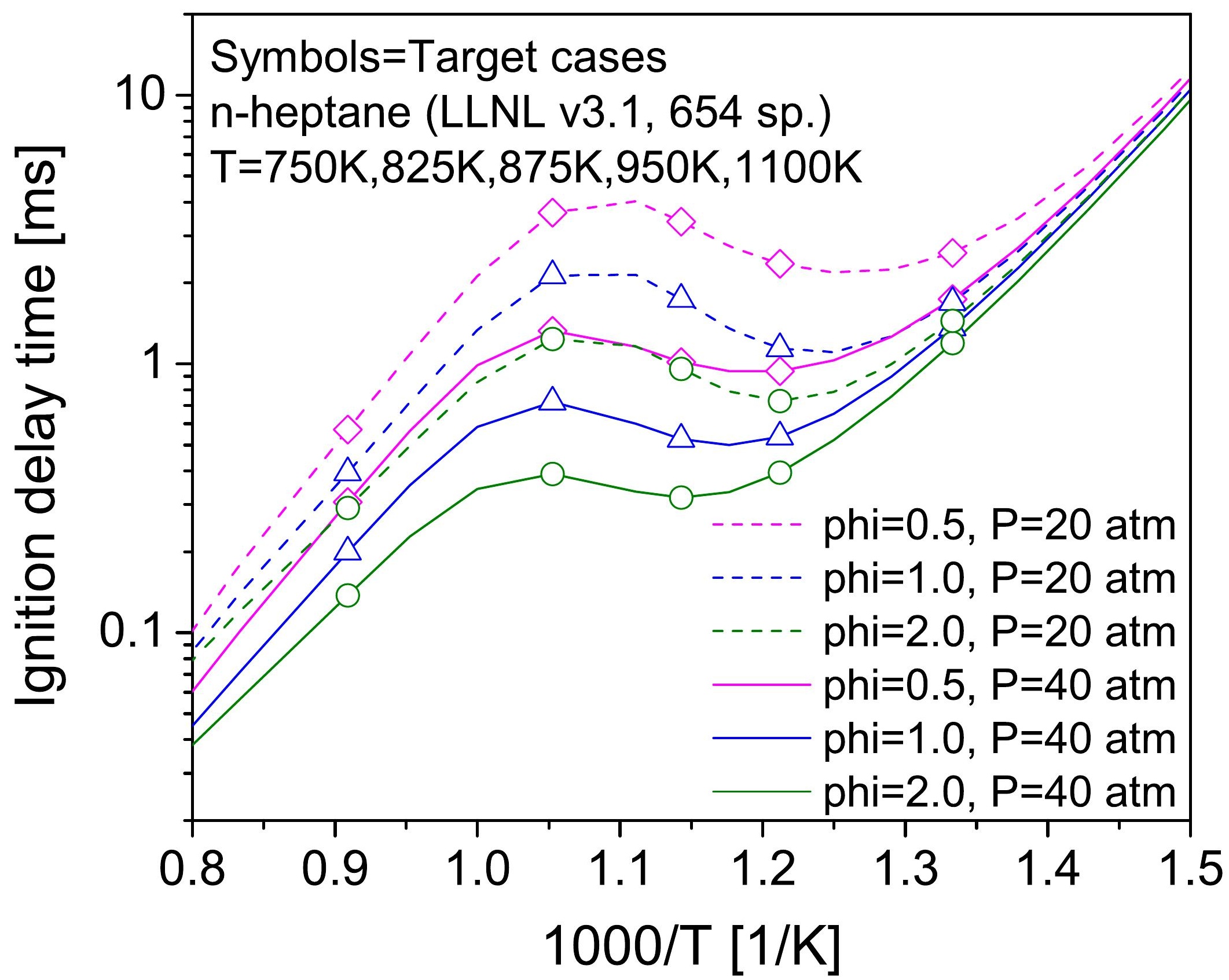


Figure 7

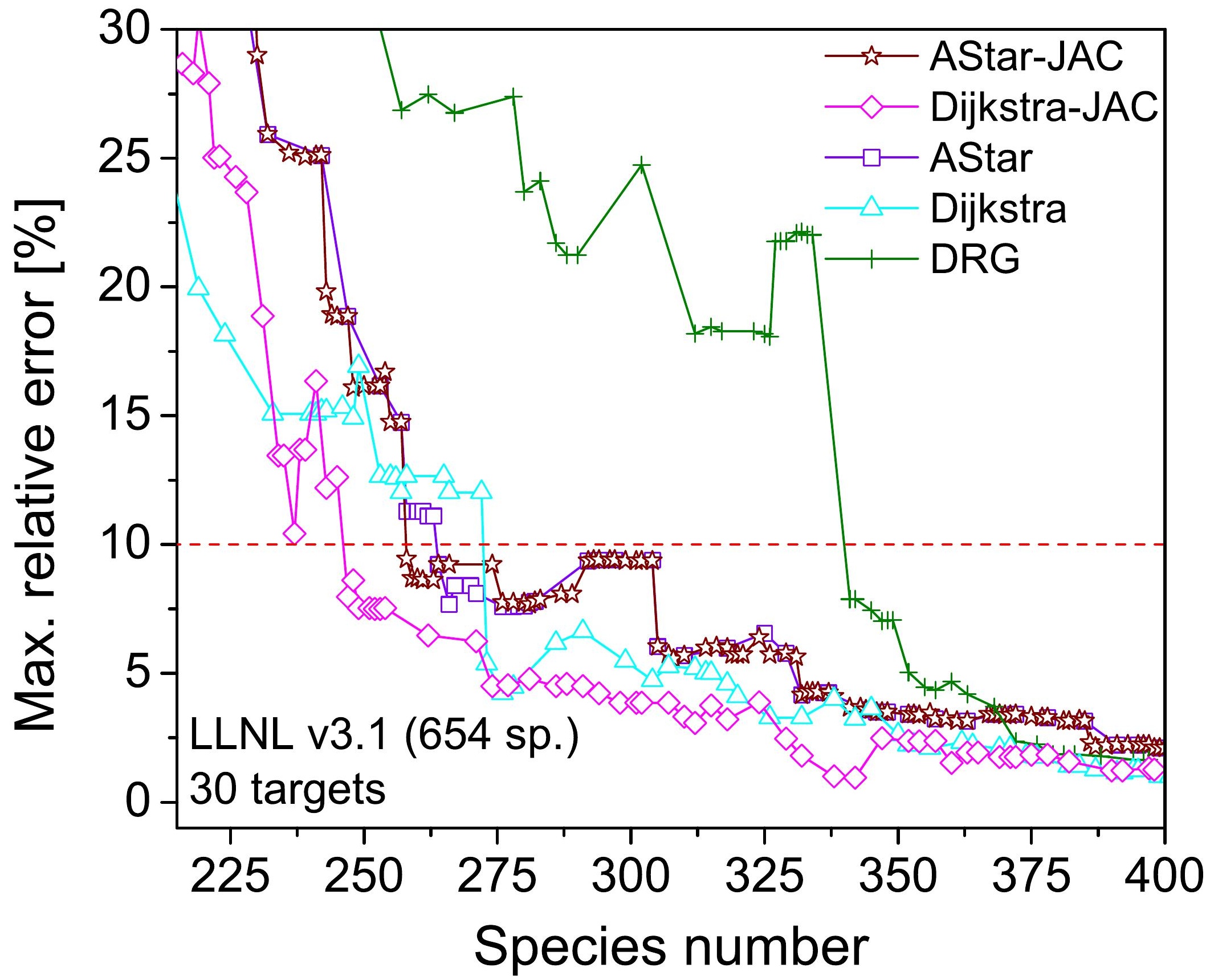




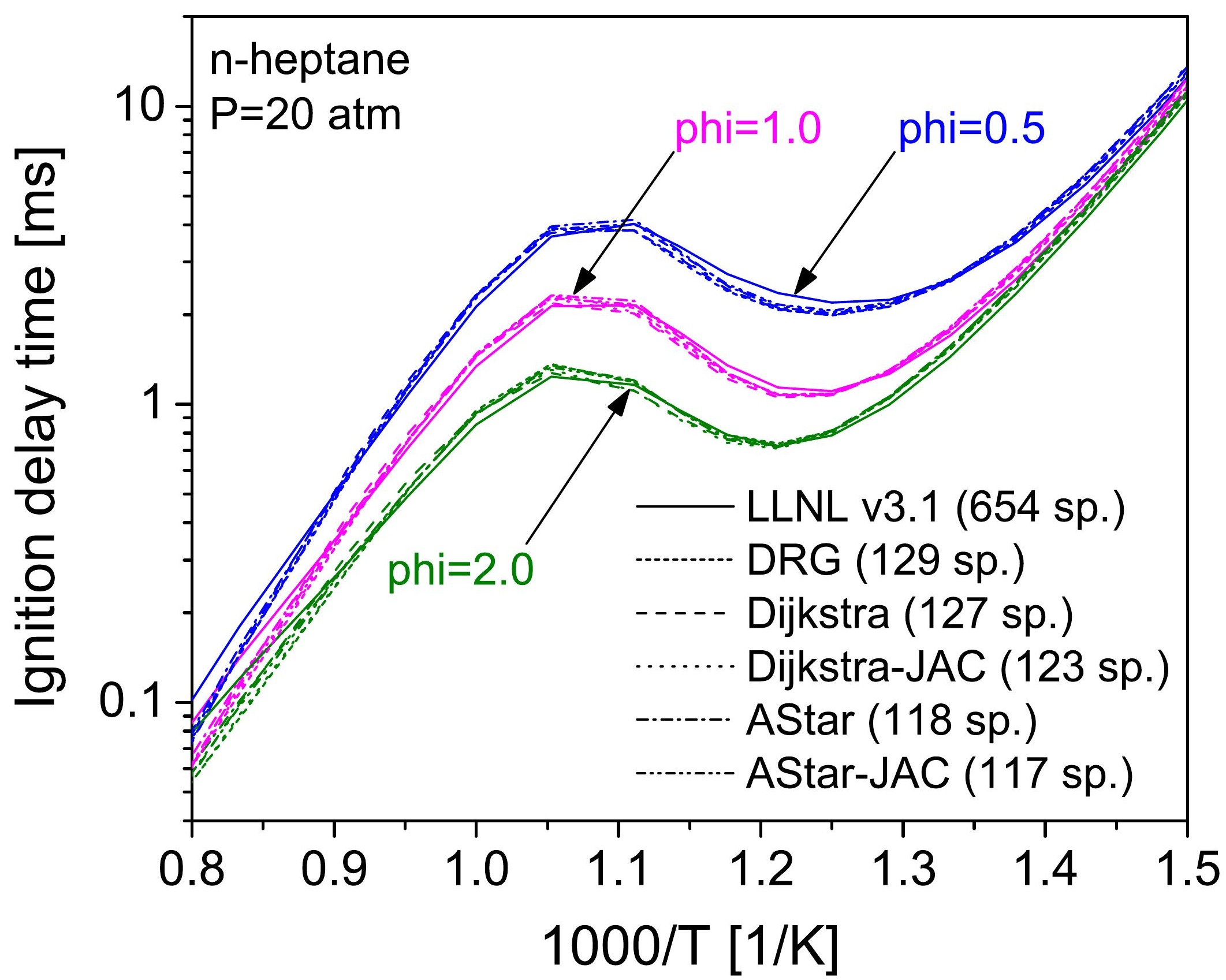




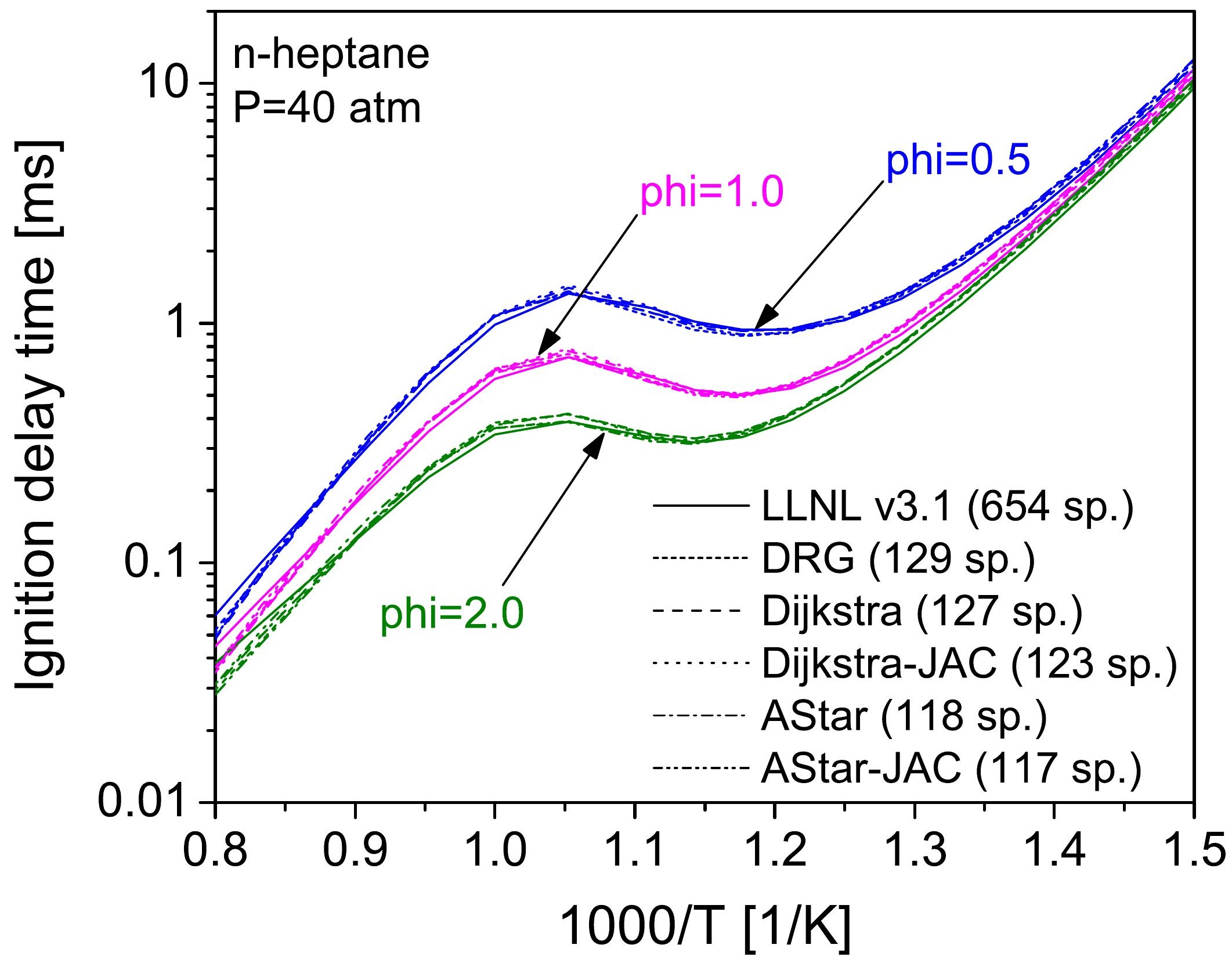




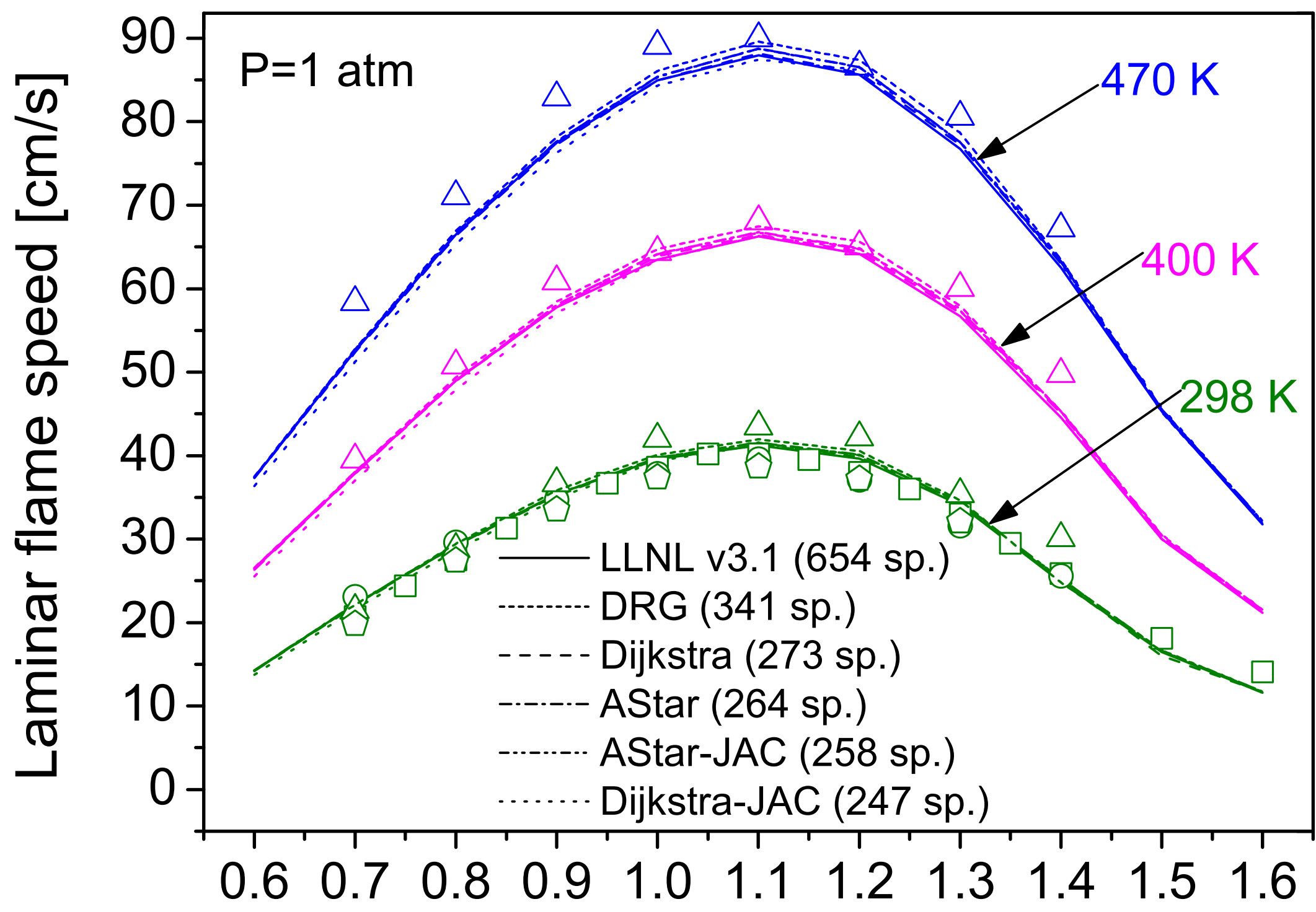

\section{Equivalence ratio}




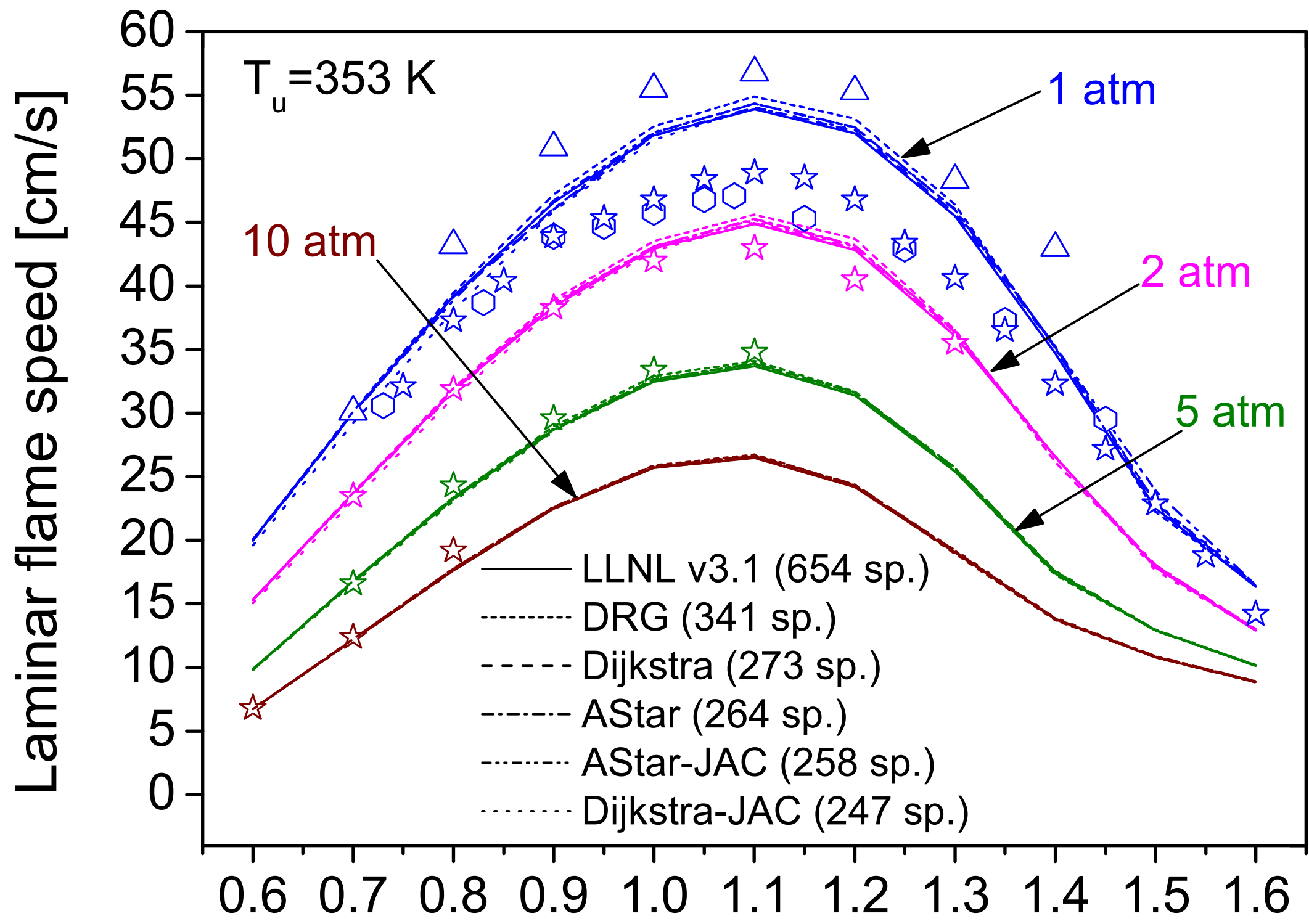

\section{Equivalence ratio}


Figure 1. A 5-node directed graph with the DIC value for each edge.

Figure 2. Searching processes and results of the Dijkstra's algorithm through a DRGEP-based method.

Figure 3. Searching process and result of the AStar algorithm through a DRGEP-based method.

Figure 4. The maximum relative error against species number of skeletal mechanism developed by various graph search methods under high temperature conditions. The detailed mechanism is the GRI-3.0 mechanism [28] with 53 species.

Figure 5. Comparison of methane ignition delay time between the detailed mechanism and the final 19-species skeletal mechanisms.

Figure 6. Thirty n-heptane target cases selected for LLNL v3.1 mechanism reduction with NTC regions.

Figure 7. The maximum relative error against species number of skeletal mechanism developed by various graph search methods in NTC regions.

Figure 8. Comparison of n-heptane ignition delay time between the detailed mechanism and the final skeletal mechanisms for equivalence ratio of $0.5,1.0$ and 2.0 under the pressure of 20 atm.

Figure 9. Comparison of ignition delay time between the detailed mechanism and the final skeletal mechanisms for equivalence ratio of $0.5,1.0$ and 2.0 under the pressure of $40 \mathrm{~atm}$.

Figure 10. Comparison of laminar flame speed between the detailed mechanism and skeletal mechanisms generated by DRGEP-based methods for unburned gas temperature of $298 \mathrm{~K}, 400 \mathrm{~K}$ and $470 \mathrm{~K}$ under the pressure of 1 atm. Symbols are experimental data: triangle: Kumar et al. [38]; square: Davis and Law [39]; circle: Huang et al. [40]; pentagon: Sileghem et al. [41].

Figure 11. Comparison of laminar flame speed between the detailed mechanism and skeletal mechanisms generated by DRGEP-based methods for pressure of 1 atm, 2 atm and 5 atm under the unburned gas temperature 
of 353 K. Symbols are experimental data: triangle: Kumar et al. [38]; hexagon: Smallbone et al. [42]; star: Kelley et al. [43]. 